\title{
Solar wind velocity at solar maximum: A search for latitudinal effects
}

\author{
B. Bavassano, R. D’Amicis, and R. Bruno \\ Istituto di Fisica dello Spazio Interplanetario (C.N.R.), Roma, Italy \\ Received: 5 February 2004 - Revised: 29 June 2004 - Accepted: 2 July 2004 - Published: 3 November 2004
}

\begin{abstract}
Observations by Ulysses during its second out-ofecliptic orbit have shown that near the solar activity maximum the solar wind appears as a highly variable flow at all heliolatitudes. In the present study Ulysses data from polar latitudes are compared to contemporary ACE data in the ecliptic plane to search for the presence of latitudinal effects in the large-scale structure of the solar wind velocity. The investigated period roughly covers the Sun's magnetic polarity reversal. The Ulysses-ACE comparison is performed through a multi-scale statistical analysis of the velocity fluctuations at scales from 1 to 64 days. The results indicate that, from a statistical point of view, the character of the wind velocity structure does not appear to change remarkably with latitude. It is likely that this result is characteristic of the particular phase of the solar magnetic cycle.
\end{abstract}

Key words. Interplanetary physics (interplanetary magnetic fields; solar wind plasma; sources of the solar wind)

\section{Introduction}

The three-dimensional structure of the solar wind observed by Ulysses at high solar activity during its second out-ofecliptic orbit is completely different from that seen at low activity along the first orbit (McComas et al., 2003). In fact, in the latter case the solar wind is characterized by a bimodal structure (McComas et al., 1998), with a steady, fast wind at high latitudes and a slower and more variable wind at low latitudes. Conversely, at high solar activity highly variable flows are observed at all latitudes and the wind structure appears to be a complicated mixture of flows coming from a variety of sources (McComas et al., 2002a,b, 2003; Neugebauer et al., 2002; Fujiki et al., 2003; Riley et al., 2003).

This state with variable flows at all heliographic latitudes is a short-lived feature of the heliosphere. After the reversal of the solar magnetic field (Jones et al., 2003), a recovery of the wind's bimodal structure begins quite soon. Ulysses observations along the northern leg of the second orbit already

Correspondence to: B. Bavassano

(bavassano@ifsi.rm.cnr.it) indicate a robust return of the high-latitude fast wind (McComas et al., 2002b). The only opportunity to investigate the properties of the high-latitude solar wind around solar maximum before the recovery of a bimodal structure is offered by the Ulysses plasma measurements along the second orbit's southern leg (it is worth recalling that in its out-of-ecliptic orbits Ulysses is first travelling in the Southern Hemisphere, then in the Northern one, with a fast latitudinal scan from south to north through the perihelion).

In the present study we will analyse a subset of Ulysses data from this southern polar phase, more precisely the time interval during which the spacecraft moves from $50^{\circ} \mathrm{S}$ to the highest latitude $\left(80.2^{\circ} \mathrm{S}\right)$ and then back to $50^{\circ} \mathrm{S}$. We will perform a comparison with data collected at the same time by ACE, which is orbiting in the ecliptic plane around the Sun-Earth L1 libration point $(\sim 0.01 \mathrm{AU}$, astronomical unit, sunward of the Earth). Our aim is that of looking for the presence of latitudinal trends in the large-scale structure of the solar wind velocity near the solar activity maximum. While a latitudinally ordered structure, as that observed at low solar activity, is surely absent, it might well be that less obvious latitudinal trends are present in the velocity pattern. Our Ulysses-ACE comparison is based on a statistical approach, a complementary view with respect to studies such as that by Elliott et al. (2003), which was based on the use of a deterministic model.

\section{Data Analysis}

The data used in the present study are daily averages of the solar wind velocity magnitude ${ }^{\vee}$ as measured by the SWOOPS and SWEPAM plasma analyzers aboard Ulysses and ACE, respectively (for both experiments D. J. McComas is the principal investigator). As mentioned above, the analysed Ulysses data are from southern latitudes poleward of $50^{\circ} \mathrm{S}$, as displayed in Fig. 1. Here at the top we give a polar plot of versus the heliographic latitude for years 1998 to 2002. The angular sector delimited by thick lines indicates the investigated interval, from day 88 (2000) to day 63 (2001). It just corresponds to a maximum of the solar activity cycle, as evidenced by the sunspot numbers shown 


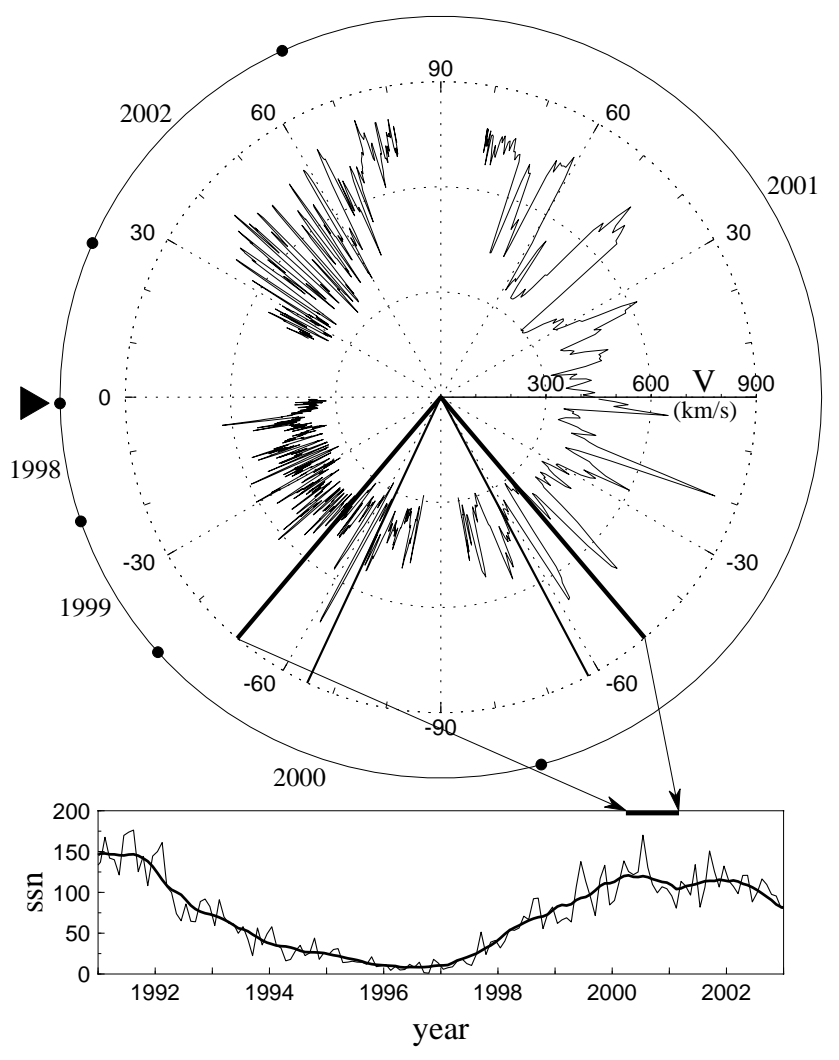

Fig. 1. A polar plot of the solar wind velocity ${ }^{`}$ vs. heliographic latitude is shown at the top for years 1998 to 2002. Dots along the outermost circle mark the beginning of each year, with time increasing anticlockwise from the leftmost dot (see arrowhead). The bottom panel gives solar sunspot numbers (ssn) in terms of monthly and smoothed values (thin and thick line, respectively) for a 12-year interval starting in 1991. The angular sector indicated by thick lines in the top plot and the thick segment in the bottom plot identify the analysed interval. A narrower sector delimited by lighter lines identifies a very-high-latitude subinterval, see text.

in the bottom plot (see the thick segment indicated by arrows). Moreover, Ulysses observations from the latitudinal scan around perihelion (McComas et al., 2002b) indicate that the polarity reversal of the solar magnetic field occurs from early 2000 to early 2001 (Jones et al., 2003), namely just during the time interval investigated here. It should also be mentioned that this same interval has already been the object of a study by McComas et al. (2002a) from the point of view of the wind solar sources.

In Fig. 2 the wind velocity ${ }^{2}$ and the spacecraft coordinates (heliocentric distance ${ }^{\circ}$ and heliographic latitude $\lambda$ ) are plotted versus time for the investigated interval. The interval is thirteen solar rotations long, as seen by Ulysses, with ${ }^{\circ}$ decreasing from 3.76 to $1.62 \mathrm{AU}$ and $\lambda$ varying from $-49.8^{\circ}$ to $-80.2^{\circ}$ and again to $-49.8^{\circ}$. The velocity profile shown in Fig. 2 is quite variable, with slow flows close to $\sim 300 \mathrm{~km} / \mathrm{s}$ and peaks at $\sim 700 \mathrm{~km} / \mathrm{s}$. As indicated by McComas et al. (2002a), the velocity peaks observed during this interval generally are related to flows originating from small coronal holes. A remarkable feature in Fig. 2 is that

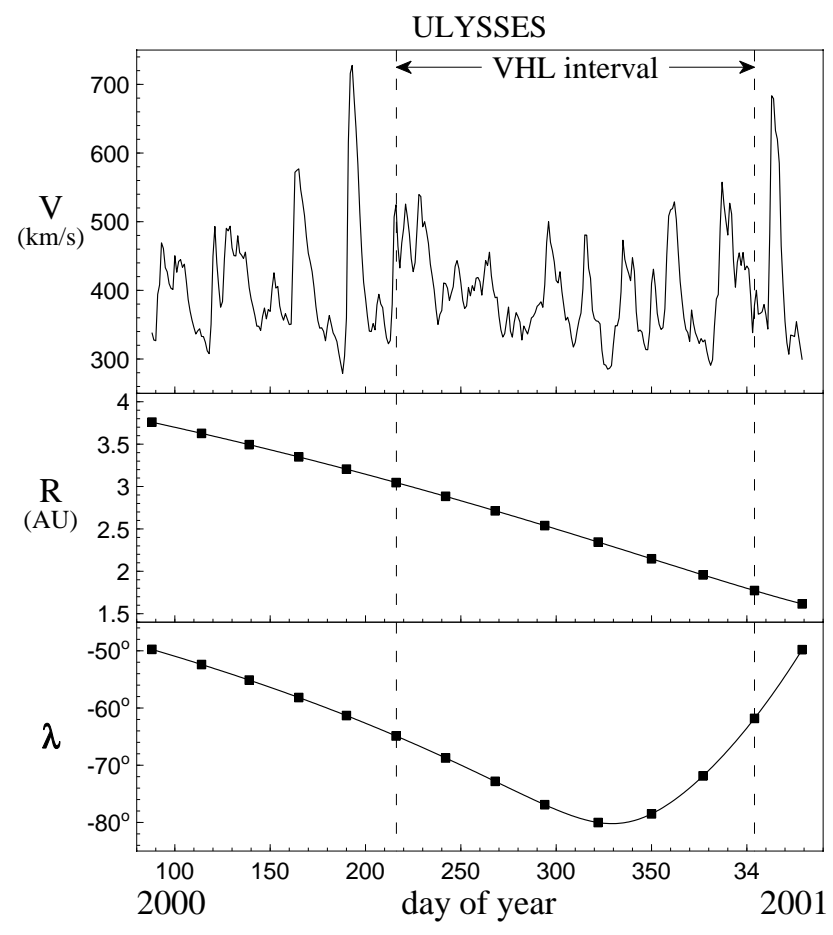

Fig. 2. The solar wind velocity ${ }^{`}$ and the Ulysses heliocentric distance ${ }^{\circ}$ and heliographic latitude $\lambda$ are plotted vs. time for the investigated interval. Dashed lines delimit an interval at very high latitudes (VHL), where the ${ }^{`}$ curve does not exhibit prominent peaks.

$\checkmark$ does not exhibit any major peak for quite a long period of time (approximately from day 200, 2000 to 45, 2001), corresponding roughly to latitudes poleward of $60^{\circ} \mathrm{S}$. This peculiarity has led us to focus our study on this subinterval at higher latitudes. Thus, in addition to the full interval of thirteen solar rotations mentioned above, in the following we will also analyse a shorter interval spanning from day 216 , $2000\left(\stackrel{\circ}{=} 3.05 \mathrm{AU}, \lambda=-64.89^{\circ}\right)$ to day $37,2001 \stackrel{\circ}{=} 1.78 \mathrm{AU}$, $\lambda=-62.25^{\circ}$ ), with a total duration of seven rotations. It is highlighted in Fig. 2 by dashed lines and labelled as VHL (very-high-latitude) interval. In the top plot of Fig. 1 the VHL interval corresponds to the angular sector delimited by light lines.

A multi-scale statistical analysis of the velocity variations (e.g. see Burlaga and Forman, 2002; Burlaga et al., 2003) has been used to characterize the structure of the solar wind at time scales above $\sim 1$ day. The procedure is quite simple. First, from daily averages of the wind velocity magnitude, time series of the velocity differences at given time lags $\tau$ have been obtained. Choosing $\tau$ equal to $2^{n}$ days for $n=0,1,2,3,4,5$, and 6 , seven time series $d V n \equiv d V n(t)=V\left(t+2^{n}\right)-V(t)$ have been derived. Then, for each time series (i.e. each time lag), the occurrence frequency distribution of the $d V n(t)$ population and the corresponding values of mean, standard deviation, skewness, and kurtosis, have been computed. The values at the different lags of these statistical quantities provide an overview of the basic features of the solar wind velocity structure. 


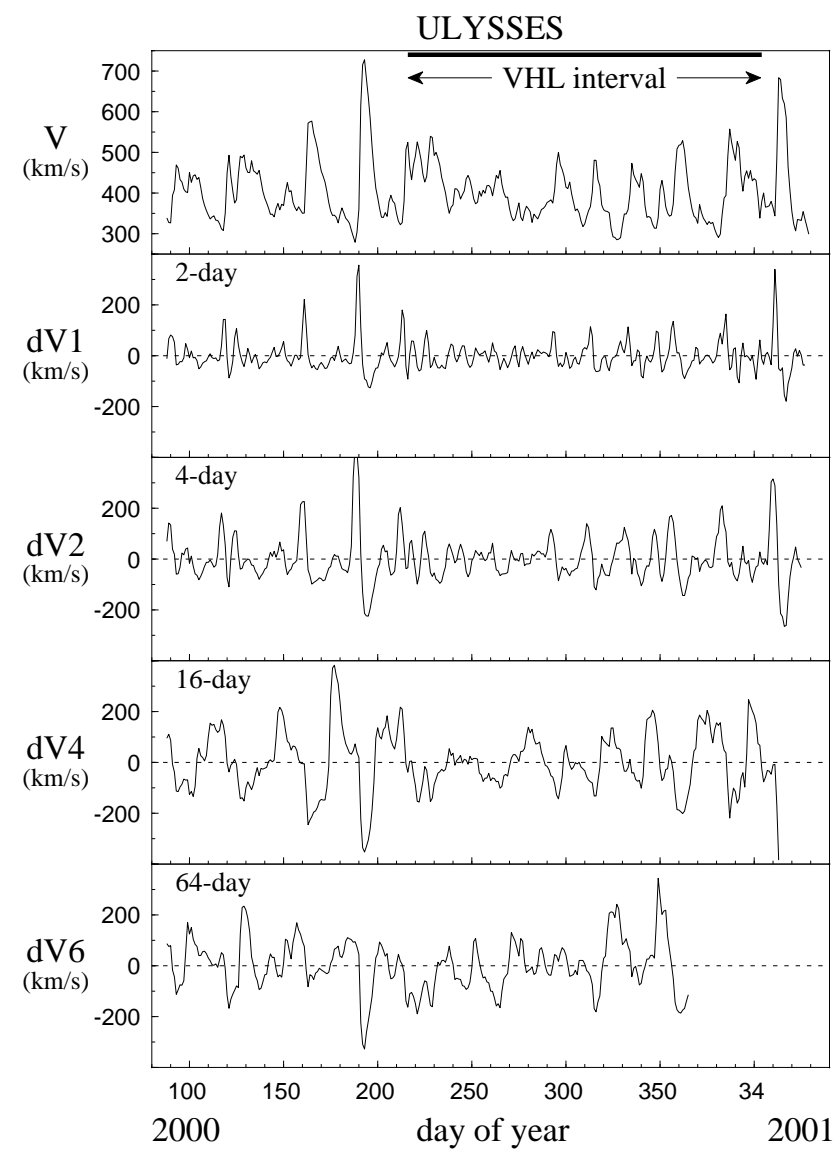

Fig. 3. From top to bottom: solar wind velocity ${ }^{`}$ and velocity differences $d V 1, d V 2, d V 4$, and $d V 6$ (at time lags of 2, 4, 16, and 64 days, respectively) versus time for the analysed Ulysses data. A bar on top indicates the VHL (Very-High-Latitude) interval.

As is well known, the skewness measures the asymmetry of a distribution and the kurtosis measures its peakedness (relative to a Gaussian distribution). For a Gaussian distribution the skewness is obviously 0 , while the kurtosis, as defined by the ratio of the fourth to the squared second moment of the distribution, has a value of 3 . The definition used here gives an unbiased estimate of the kurtosis (essentially with subtraction of the factor 3), thus in the present paper the kurtosis is 0 for a Gaussian distribution.

\section{Velocity differences and frequency distributions}

\subsection{Ulysses observations}

The velocity differences $d V 1, d V 2, d V 4$, and $d V 6$ (at time lags of 2, 4, 16, and 64 days, respectively) for the analysed Ulysses data are plotted versus time in Fig. 3 (second to fifth panel from top). In the top panel the ${ }^{\vee}$ daily averages, already shown in Fig. 2, are reported for easier reference.

At small scales the velocity differences are relatively small and spiky (e.g. see the $d V 1$ curve), with the exception of values coming from sharp gradients of $y$ At larger scales the

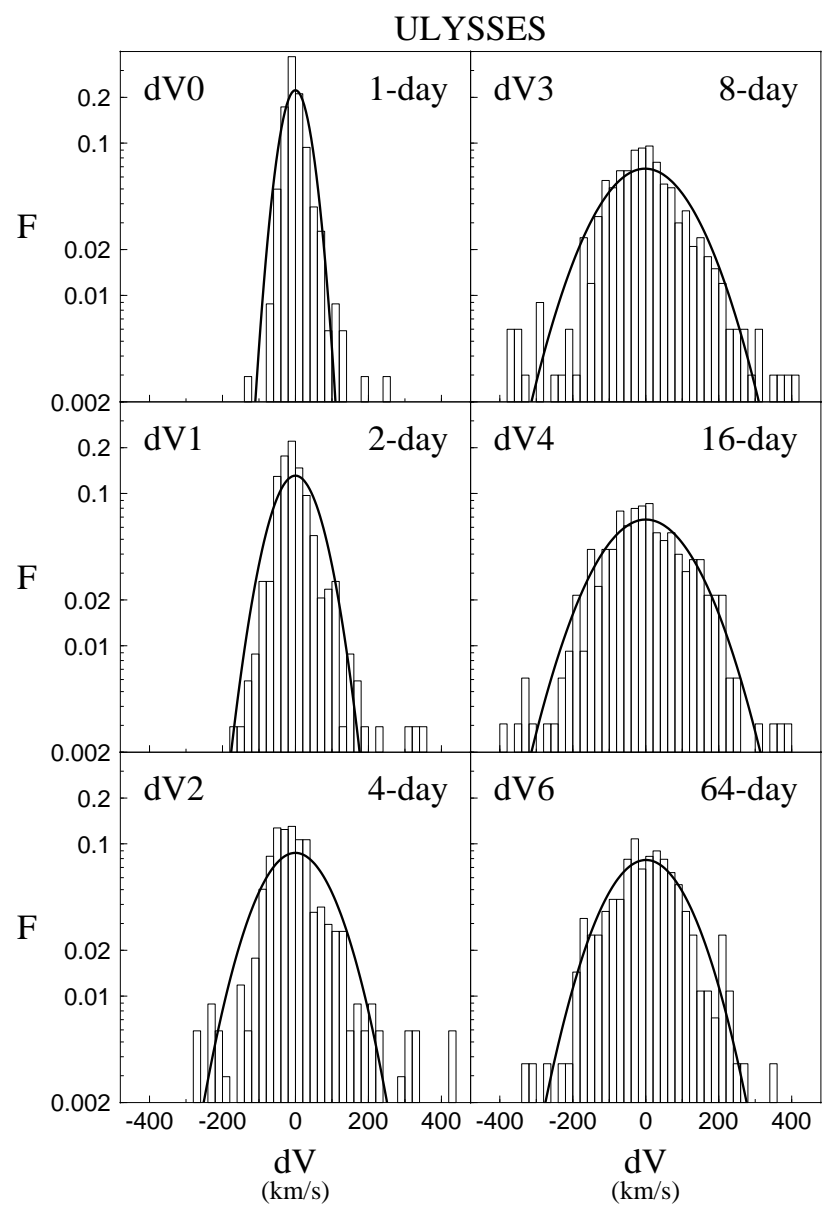

Fig. 4. The occurrence frequency $F$ (in a logarithmic scale) of the Ulysses velocity differences $d V 0, d V 1, d V 2, d V 3, d V 4$, and $d V 6$ is shown by histograms in the different panels. The solid curves give Gaussian distributions as computed from the observed values of mean and standard deviation.

curves exhibit smoother variations of larger amplitude. This is similar to the findings of Burlaga et al. (2003).

The occurrence frequency $\mathrm{F}$ of the Ulysses velocity differences $d V 0, d V 1, d V 2, d V 3, d V 4$, and $d V 6$ (corresponding to $1-, 2-, 4-, 8-, 16-, 64-$ day time lags) is shown by histograms in the panels of Fig. 4 . The curves indicate Gaussian distributions as obtained from the observed values of mean and standard deviation (i.e. they are not the result of a fit procedure). It is clearly seen that the distribution width increases going from $1-$ to 8 -day lags, then remains almost constant. At a first glance the histograms appear qualitatively closer to a Gaussian curve at large scales. These points will be thoroughly discussed in Sect. 4.

\subsection{ACE observations}

As mentioned in the Introduction, our analysis is based on the comparison between Ulysses data from the Southern Hemisphere (latitudes poleward of $50^{\circ} \mathrm{S}$ ) and contemporary data by ACE on the ecliptic plane. The analysed ACE interval spans from day 77, 2000 to day 61, 2001. As for Ulysses, 


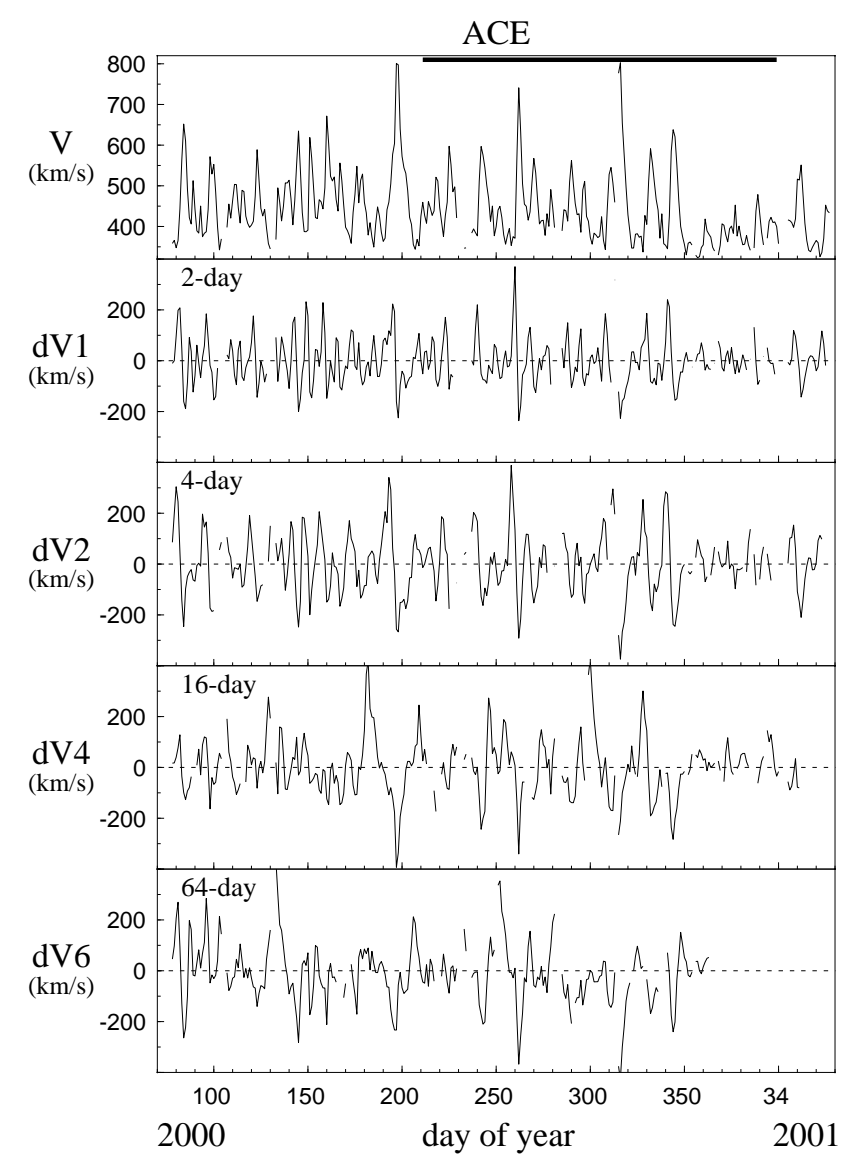

Fig. 5. Solar wind velocity ` and velocity differences $d V 1, d V 2$, $d V 4$, and $d V 6$ for the analysed ACE interval, in the same format of Fig. 3. A bar on top indicates the ACE interval that corresponds to the Ulysses VHL interval.

this interval has a total length of thirteen solar rotations. The time changes with respect to the Ulysses data base are to account for a slightly different duration of the solar rotation as seen by ACE (in a halo orbit at L1) and by Ulysses (in a deep space orbit). Moreover, a time delay to allow for solar wind propagation from ACE (at $1 \mathrm{AU}$ ) to Ulysses distances (3.76 to $1.62 \mathrm{AU}$ ) has been taken into account, although this kind of approach may be questionable for the case of largely separated spacecraft in a high-solar-activity wind (e.g. see Elliot et al., 2003; Riley et al., 2003).

The solar wind velocity (daily averages) for the analysed ACE interval is shown in the top panel of Fig. 5. Similarly to the Ulysses observations, the ACE velocity profile is quite variable. A bar on top indicates the ACE interval (days 211, 2000 to 33, 2001) that corresponds to the Ulysses VHL interval. As in Fig. 3, the second to fifth panels (from top) give the velocity differences $d V 1, d V 2, d V 4$, and $d V 6$. At a first glance, the curves at small lags seem to indicate an enhanced variability of the ACE data as compared to Ulysses data.

The frequency histograms of the ACE velocity differences are given in Fig. 6, in the same format of Fig. 4. As for Ulysses, going from $1-$ to 8 -day lags the distribution width

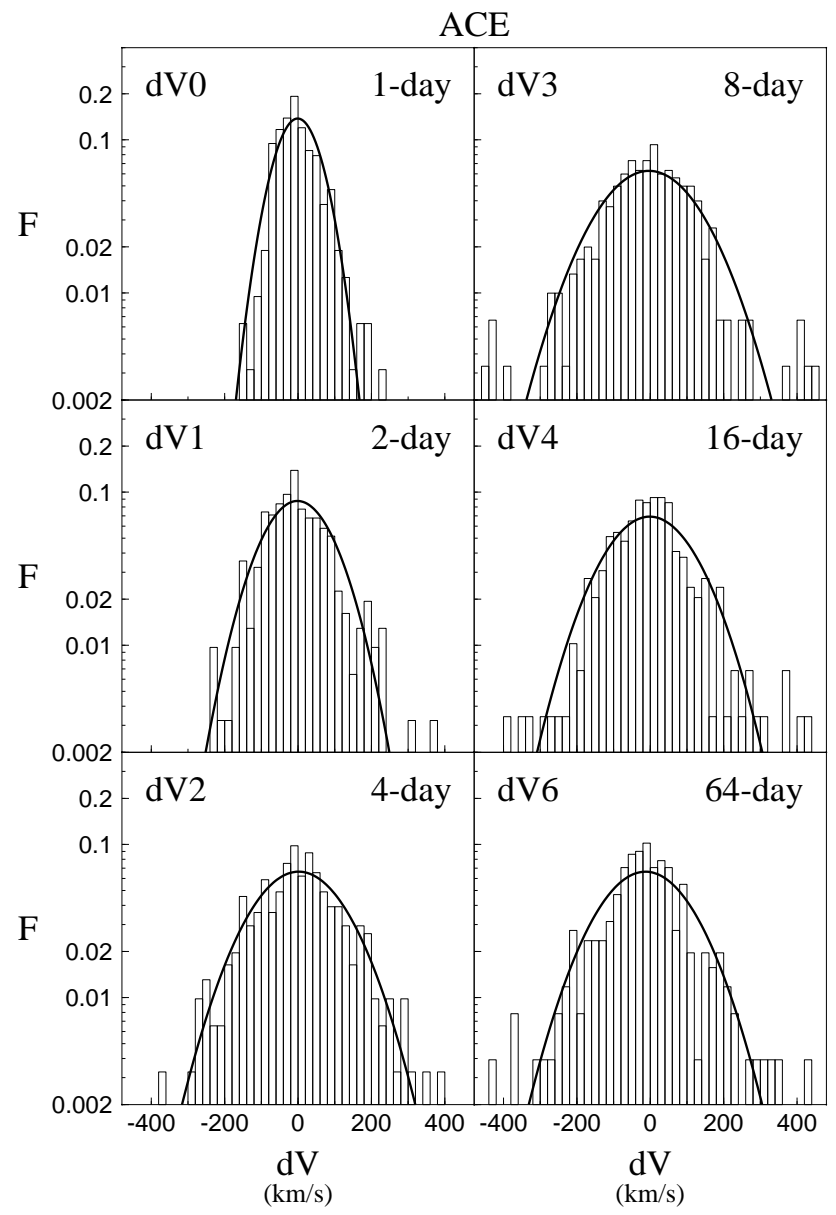

Fig. 6. Frequency histograms for the ACE velocity differences, in the same format of Fig. 3.

increases, then (i.e. at larger lags) this trend disappears. Moreover, it is easily seen that at small lags the ACE histograms are wider than those for Ulysses.

\section{Moments}

As already mentioned, a simple way to get an overall description of the $d V n$ distribution properties and of their dependence upon scale (i.e. the time lag $\tau$ ) is that of looking at the behaviour of their moments.

In Fig. 7 the dependence on $\tau$ of mean, standard deviation, skewness, and kurtosis is shown separately for Ulysses (solid lines) and ACE (dashed lines) data. Regarding the mean (top panel), it is seen to be close to zero at all scales for Ulysses. For ACE this does not hold at time lags of 32 and 64 days. However, the difference from zero, in absolute value, does not go beyond $\sim 10 \%$ of the standard deviation. The standard deviation (second panel from top) roughly ranges from 35 to $120 \mathrm{~km} / \mathrm{s}$ for Ulysses and from 60 to $130 \mathrm{~km} / \mathrm{s}$ for ACE. With the exception of $\tau=16$, the standard deviation is always higher for ACE data. Skewness and kurtosis (third and fourth panels) indicate a close-to-Gaussian behaviour for ACE at 


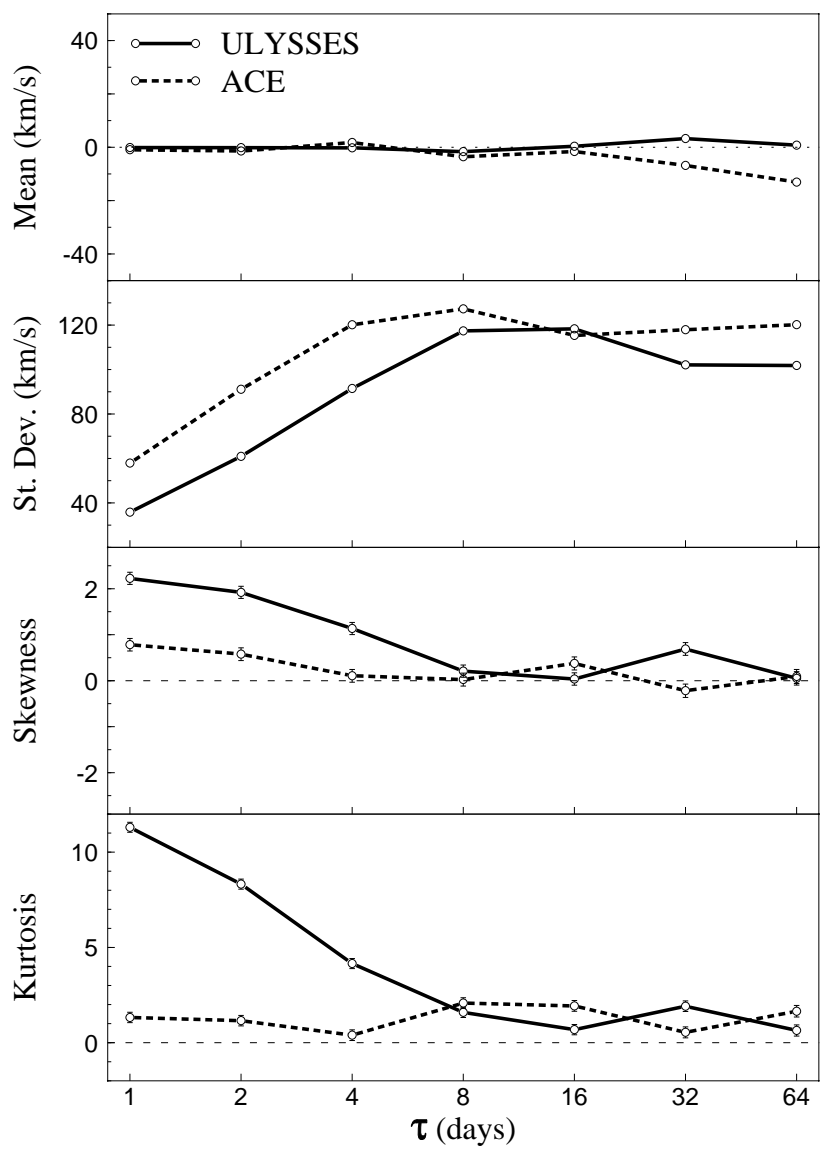

Fig. 7. The dependence of mean, standard deviation, skewness, and kurtosis on the time lag $\tau$ is shown separately for Ulysses (solid lines) and ACE (dashed lines) data. Error bars are given for skewness and kurtosis (in some cases errors are smaller than the marker size).

all scales. In contrast, non-Gaussian features come out for Ulysses at $\tau<8$, with a kurtosis value of $\sim 11$ at the smallest scale.

The presence of non-Gaussian features in the Ulysses distributions is a relevant point and needs to be carefully investigated. From $d V 1, d V 2$ and $d V 3$ Ulysses data plotted in Fig. 3 it clearly appears that the values outside the core of the distribution mainly come from the two major ${ }^{`}$ peaks (top panel) near days 193 (2000) and 47 (2001). The VHL interval, covering the Ulysses trajectory phase poleward of $\sim 65^{\circ}$, falls just between these two peaks. From Fig. 2 it can be argued that the solar wind conditions above the Sun's polar regions are better described by data from the VHL interval, rather than from the entire examined interval. Then, we have focused our analysis on the VHL interval of Ulysses data and on the corresponding ACE data interval (see Fig. 5 on top). The new values of mean, standard deviation, skewness, and kurtosis are plotted in Fig. 8. It clearly appears that at all scales the Ulysses values for skewness and kurtosis are now close to zero (and close to the corresponding ACE values). Thus, the above indication about non-Gaussian features does not hold for VHL data.

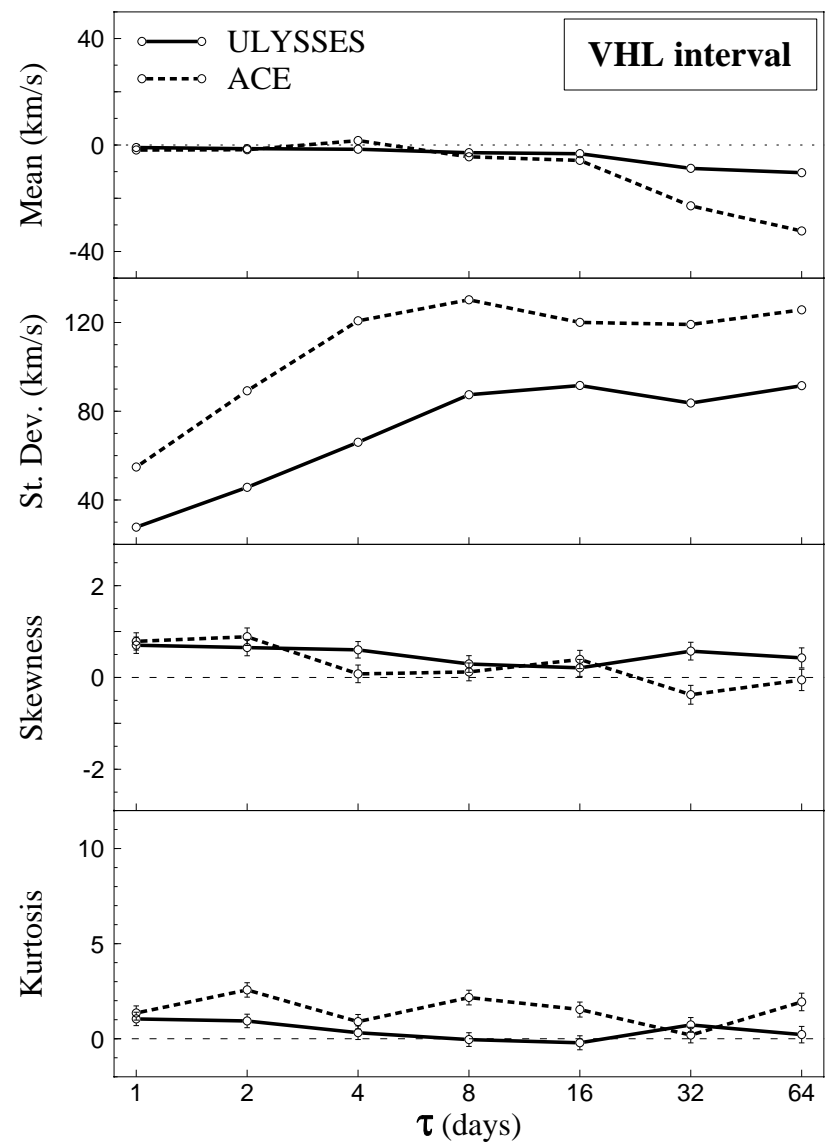

Fig. 8. Mean, standard deviation, skewness, and kurtosis vs. time lag $\tau$ for the VHL interval, in the same format of Fig. 7 .

Regarding the mean and standard deviation, Fig. 8 confirms that mean values different from zero come out at large $\tau$ (up to $25 \%$ of the standard deviation for ACE, perhaps an effect of data gaps) and that at all scales the standard deviation values for ACE are higher than those for Ulysses. Since Ulysses and ACE measurements come from different radial distances, the question arises if the standard deviation decrease at Ulysses may be ascribed to a radial effect. This point is addressed by Fig. 9. Here we plot the $d V 1$ standard deviations for Ulysses (filled triangles, down for the whole data interval and up for the VHL interval), ACE (triangles, down and up as for Ulysses), and Wind (filled square). Average radial distances are used for Ulysses values (2.74 and 2.43 AU for the whole data set and the VHL interval, respectively). The Wind-95 value refers to a thirteen solar rotation interval in 1995 (days 12 to 362). The solid curve shown in the figure is from Burlaga et al. (2003). It has been derived by applying a model developed by Wang et al. (2002) (see more references in the paper by Burlaga et al.) to describe the solar wind evolution in the outer heliosphere. Using 1995 Wind data as input to the model at 1 AU, Burlaga et al. (2003) determined the velocity structure from 5 to $95 \mathrm{AU}$ at steps of $5 \mathrm{AU}$ and computed the distribution moments for $d V 1$. The curve is an exponential fit to the standard deviation values ob- 


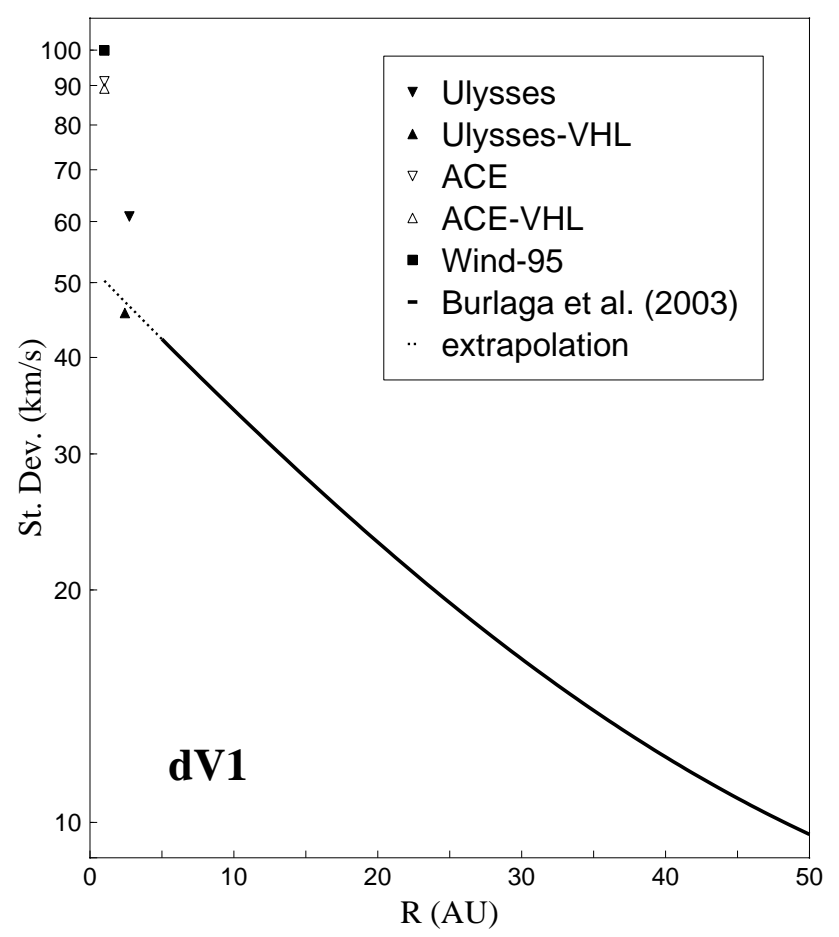

Fig. 9. The standard deviation as measured by Ulysses and by nearEarth spacecraft (ACE and Wind). The solid curve is from Burlaga et al. (2003), dots indicate an extrapolation to $1 \mathrm{AU}$.

tained with this procedure. It is immediately apparent from Fig. 9 that the ACE and Wind standard deviations (triangles and filled square) are much higher than the extrapolation to $1 \mathrm{AU}$ (dotted curve) of the 5-95 AU fit. Thus, from 1 to $5 \mathrm{AU}$ the standard deviation decrease has to be much faster than beyond $5 \mathrm{AU}$. The Ulysses standard deviations (filled triangles) appear to be in reasonable agreement with this ecliptic radial trend. Then, the outcome of a lower standard deviation at Ulysses with respect to ACE is probably a radial rather than a latitudinal effect. It must be stressed, however, that all this holds only for $d V 1$. The extension to other scales is just a hypothesis. A last comment about Fig. 9, though marginal for the present paper, is that the difference between ACE and Wind standard deviations at $1 \mathrm{AU}$ is small, though they refer to periods at completely different phases of the solar cycle (see, in the bottom plot of Fig. 1, the sunspot number for 1995 and 2000-2001 periods). In other words, the solar activity does not appear to affect the solar wind variability as measured by $d V 1$.

\section{Conclusions}

Ulysses data from southern latitudes greater than $50^{\circ}$ have been compared to contemporary ACE data in the ecliptic plane to search for the presence of latitudinal effects in the large-scale structure of the solar wind velocity. The study refers to high solar activity conditions, when the solar wind appears as a highly variable flow at all latitudes. The compar- ison is performed by using a multi-scale statistical analysis of the velocity differences at scales from 1 to 64 days.

The skewness and kurtosis behaviours for the whole interval investigated indicate a close-to-Gaussian behaviour for ACE at all the scales. In contrast, non-Gaussian features come out for Ulysses at the small scales $(\tau<8$ days). But, if we focus the analysis on the VHL (Very-High-Latitude) interval, this last result does not appear to be confirmed. In fact, for Ulysses VHL data, both skewness and kurtosis are close to 0 (and close to the ACE values) at all the scales. Solar wind conditions above the Sun's poles appear better described by data from the VHL interval rather than from the whole interval examined. Then, we are led to conclude that at the examined scales the distributions of the velocity differences have a Gaussian character for both polar and ecliptic solar wind.

Differences between Ulysses and ACE are observed for the standard deviation level, with ACE values higher than those for Ulysses. We have shown that the Ulysses standard deviations are in reasonable agreement with the overall radial variation observed in the ecliptic plane. Then, the UlyssesACE differences probably are a radial rather than latitudinal effect.

In conclusion, from a statistical point of view, the character of the solar wind velocity structure at large scales around solar maximum does not appear to change remarkably with latitude. It should be recalled that the time interval investigated here roughly corresponds to that of the polarity reversal of the solar magnetic field (Jones et al., 2003). Under such conditions it is not surprising that latitude does not play any relevant role in shaping the solar wind. However, as already underlined in the Introduction, the investigated interval is a unique, short-lived period in the context of the entire solar activity cycle. During most of the cycle the wind velocity exhibits a well developed latitudinal pattern.

As a final remark it should be stressed again that our conclusions belong to the statistical domain. When the UlyssesACE comparison is done for specific individual structures (Elliott et al., 2003), it comes out that at latitude separations above $\sim 30^{\circ}$ no correlation is left.

Acknowledgements. The use of data from the Ulysses/SWOOPS and ACE/SWEPAM plasma analyzers (principal investigator D. J. McComas, Southwest Research Institute, San Antonio, Texas, USA) is gratefully acknowledged. The data have been obtained through the NASA World Data Center A for Rockets and Satellites (Goddard Space Flight Center, Greenbelt, Maryland, USA). Ruth Skoug has kindly made available ACE data for the Bastilleday event. Solar sunspot data are from the World Data Center for the Sunspot Index, Royal Observatory of Belgium (Brussels, Belgium). The present work has been supported by the Italian Space Agency (ASI) under contract IR/064.

Topical Editor R. Forsyth thanks two referees for their help in evaluating this paper. 


\section{References}

Burlaga, L.F. and Forman, M. A.: Large-scale speed fluctuations at 1 AU on scales from $1 \mathrm{~h}$ to $\approx 1$ year: 1999 and 1995, J. Geophys. Res., 107(A11), 1403, doi:10.1029/2002JA009271, 2002.

Burlaga, L. F., Wang, C., Richardson, J. D., and Ness, N. F.: Evolution of the multiscale statistical properties of corotating streams from 1 to 95 AU, J. Geophys. Res., 108(A7), 1305, doi:10.1029/2003JA009841, 2003.

Elliott, H. A., McComas, D. J., and Riley, P.: Latitudinal extent of large-scale structures in the solar wind, Ann. Geophys., 21, 1331-1339, 2003.

Fujiki, K., Kojima, M., Tokumaru, M., Ohmi, T., Yokobe, A., Hayashi, K., McComas, D. J., and Elliott, H. A.: How did the solar wind structure change around the solar maximum? From interplanetary scintillation observation, Ann. Geophys., 21, 12571261, 2003.

Jones, G. H., Balogh, A., and Smith, E. J.: Solar magnetic field reversal as seen at Ulysses, Geophys. Res. Lett., 30(19), 8028, doi:10.1029/2003GL017204, 2003.

McComas, D. J., Bame, S. J., Barraclough, B. L., Feldman, W. C., Funsten, H. O., Gosling, J. T., Riley, P., Skoug, R., Balogh, A., Forsyth, R., Goldstein, B. E., and Neugebauer, M.: Ulysses return to the slow solar wind, Geophys. Res. Lett., 25, 1-4, 1998.
McComas, D. J., Elliot, H. A., and von Steiger, R.: Solar wind from high-latitude coronal holes at solar maximum, Geophys. Res. Lett., 29(9), doi:10.1029/2001GL013940, 2002a.

McComas, D. J., Elliot, H. A., Gosling, J. T., Reisenfeld, D. B., Skoug, R. M., Goldstein, B. E., Neugebauer, M., and Balogh, A.: Ulysses' second fast-latitude scan: Complexity near solar maximum and the reformation of polar coronal holes, Geophys. Res. Lett., 29(9), doi:10.1029/2001GL014164, 2002b.

McComas, D. J., Elliott, H. A., Schwadron, N. A., Gosling, J. T., Skoug, R. M., and Goldstein, B. E.: The three-dimensional solar wind around solar maximum, Geophys. Res. Lett., 30(10), 1517, doi:10.1029/2003GL017136, 2003.

Neugebauer, M., Liewer, P. C., Smith, E. J., Skoug, R. M., and Zurbuchen, T. H.: Sources of the solar wind at solar activity maximum, J. Geophys. Res., 107(A12), 1488, doi:10.1029/2001JA000306, 2002.

Riley, P., Mikić, Z., and Linker, J. A.: Dynamical evolution of the inner heliosphere approaching solar activity maximum: interpreting Ulysses observations using a global MHD model, Ann. Geophys., 21, 1347-1357, 2003.

Wang, C., Richardson, J. D., and Gosling, J. T.: A numerical study of the evolution of the solar wind from Ulysses to Voyager 2, J. Geophys. Res., 105, 2337-2344, 2000. 